\title{
Protective and Supportive Garments and Bracing to Enhance Extreme Sport Performance and Injury Prevention
}

\author{
J. Nyland ${ }^{1,2}$, A. Cecil'2, R. Singh ${ }^{3}$, C. Raj Pandey ${ }^{4}$ \\ 1 University of Louisville Department of Orthopaedic Surgery, Louisville, Kentucky, USA \\ 2 Spalding University, Kosair Charities College of Health and Natural Sciences, Louisville, Kentucky USA \\ 3 Grande International Hospital Department of Physiotherapy, Kathmandu, Nepal \\ ${ }^{4}$ Grande International Hospital Department of Orthopaedic Surgery and Traumatology, Kathmandu, Nepal
}

\section{CORRESPONDING AUTHOR:}

John Nyland

Kosair Charities College of Health and

Natural Sciences, Spalding University

901 South 4th Street

Louisville, KY 40203, USA

E-mail: jnyland@spalding.edu

DOI:

10.32098/mltj.02.2020.18

LEVEL OF EVIDENCE: 5

\begin{abstract}
SUMMARY
Background. Protective and supportive trunk, limb and joint garment or brace use to enhance performance and prevent injury among extreme sport athletes is evolving. Methods. This review discusses protective and supportive trunk, limb and joint garment or brace use from the perspective of the essential mental and physical demands of extreme sports with special consideration for improving capsuloligamentous, musculotendinous, and myofascial system function.

Results. Bracing to protect or support, preserve and promote natural joint function is evolving from the use of heavier, rigid, over-constraining and poorly fitting devices to lighter, more flexible, lower profile, function-enhancing garments or braces. Therapeutic exercises that combine task-specific self-efficacy and problem-solving skill development may best optimize innovative protective and supportive trunk, limb and joint garment or brace use.

Conclusions. Through greater surface contact area and enhanced cutaneous, capsuloligamentous, musculotendinous and myofascial system mechanoreceptor function, a new evolution of protective and supportive trunk, limb and joint garments or braces may be better able to effectively facilitate more natural joint protection, proprioception/kinesthesia and dynamic stability.
\end{abstract}

\section{KEY WORDS}

Experential rebabilitation; adventure sports; therapeutic exercise.

\section{INTRODUCTION}

Given the dynamic environments in which extreme sport athletes perform, adaptability and creativity are needed when rehabilitation clinicians provide guidance about protective and supportive trunk, limb and joint garment or brace use. Similarly, rehabilitation clinician interactions should consider environmental demands, the unique needs of the rehabilitating extreme sport athletes and the specifics of essential task performance. Task performance parameters should be manipulated from physical, cognitive and environmental perspectives (1). To best refine participant judgement and decision making, it is important that the extreme sports athlete is an active participant in the learning experience (1).
Through soft tissue mobilization, massage, and stretching techniques, myofascial tissue have become a growing rehabilitation treatment intervention focus. Altered coordination, proprioception, balance, pain levels, and cramping are often associated with the deep fascia and epimysium, as they influence muscle, nerve, blood vessel, and organ functions (2). In contrast, the superficial fascia is more often associated with lymphatic and superficial vein circulation and thermoregulatory functions.

Athletes can display addiction-like behaviors (3). These behaviors often lead them to devote continually increasing time and monetary resources to perform their preferred sport sport at higher skill levels, with progressively greater 
injury risks (4). In addition to the increased injury risk associated with extreme sport participation, when these athletes are prevented from sport participation because of defective, restrictive, or poorly fitting equipment, they may suffer emotional or mental distress with powerful physiological and psychological symptoms (4). Therefore, the functionality, durability and ease of use of recommended protective and supportive garments or braces is essential to use adherence.

\section{MENTAL AND PHYSICAL DEMANDS}

The demands of climbing and abundance of both psychological and physiological data (5) makes it an intriguing model for discussing the potential rationale for supportive and protective garment and brace use. Climbing intensity increases from either greater upper limb muscle engagement or greater whole body effort (6). Increased climbing route difficulty redistributes the workload to smaller muscle groups, particularly in relationship to grip-surface reductions (6). Climbing research has focused primarily on its physical requirements, placing little emphasis on its cognitive, psychological and behavioral aspects (7). Climbing, like many other extreme sports, incorporates strong physiological and psychobehavioral links $(6,8,9)$. Climbing not only requires a profound understanding of how to recognize and interpret environmental constraints such as weather conditions (temperature, humidity, wind velocity, etc.) and rock, ice or other surface characteristics, but also requires instantaneous adaptability and highly tuned skills (10) (figure 1). In this context, when protective and supportive garments and braces are used, they must adapt instantaneously to changing environmental conditions and not negatively influence the climber's ability to perform skillful movements.

Combined mental and physical climbing demands, in combination with exercise-induced central and peripheral fatigue, increases heart rate, cortisol and lactate concentrations (8). Magiera et al (6) studied athletes under high, moderate, and low physical and psychological demand climbs. They identified lower physiological demands with climbing route familiarity and the performance of single moves of comparable difficulty. Physical exercise exerts considerable salivary cortisol concentration increases, when exercise intensity exceeds $60 \% \mathrm{VO}_{2}$ max or lasts at least $20-30 \mathrm{~min}$ (11). Cortisol levels increase within 10 minutes and reach a maximum at the $10^{\text {th }}$ to $30^{\text {th }}$ minute after stress cessation (12). Similar cortisol concentration increases have also been reported for downhill mountain biking (13). Magiera et al. (6) reported that three repetitions of a difficult climbing route with a short recovery time increased post-climbing cortisol concentrations. The highest cortisol levels were observed immediately following climb descent. Even antic- ipation of stressful experiences cause sympathetic nervous system and hypothalamic-pituitary-adrenal axis activation, resulting in cortisol release (12). Selected protective and supportive devices also should not contribute to heightened climber stress levels.

When exercise intensity exceeds the anaerobic threshold, lactate accumulates in skeletal muscle, resulting in decreased intramuscular $\mathrm{pH}$ (14). Lactate removal can be improved by increasing blood flow to other body parts and enhancing its oxidation through greater use of previously inactive muscles or other organs such as the liver (15). Intense or prolonged forearm muscle activation increased lactic acid concentrations, while aerobic metabolism across greater muscle mass improved its clearance. Blood lactate concentrations after rock climbing are lower than cycling because of the smaller total muscle mass that is exercised (16). During treadwall climbing until exhaustion, maximum blood lactate concentrations are higher $(10.2-11.1 \mathrm{mmol} / \mathrm{l})$ (17) than during actual climbing $(<6.8 \mathrm{mmol} / \mathrm{l})(18)$. The aerobic and anaerobic alactic systems are the primary energy systems used during indoor rock climbing (19). When climbing wall angles exceed vertical, grip strength decreases and blood lactic acid concentrations significantly increase (20). Magiera et al. (6) found that peak heart rate was the most sensitive mental and physical stress level and workload measurement among rock climbers. Protective and supportive devices should not restrict the circulatory function necessary for lactic acid clearance and active recovery. Seifert et al. (21) showed that more experienced climbers used more diagonal hip positions relative to the climbing wall when using holds that necessitated a side-on coordination pattern. Experienced climbers also had a larger

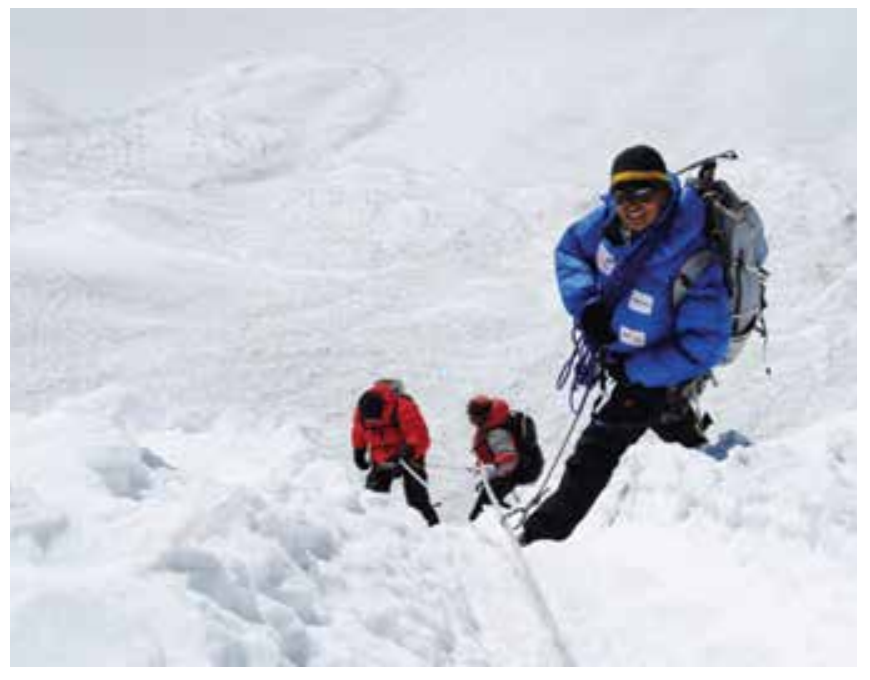

Figure 1. Environmental conditions, equipment needs. 
movement pattern repertoire so they could better adapt to constraints as climbing hold orientations changed. The learner's movement intentions are, in some ways, determined by their pre-existing coordination repertoire. The way an athlete explores and learns a new task is influenced by the number of movement solutions that they possess under an already experienced existing set of constraints (22). Implications for extreme environment climbing are that climbing walls with planned route variability can provide effective learning contexts for movement skill development when they are accompanied by opportunities that enhance environmental knowledge and decision-making skills. Safely exploring new movement coordination patterns and the complex alignment of cognitive and sensorial experiences is paramount to extreme sport performance adaptations and safety (23). While protecting or supporting a specific joint or body region, the selected brace or garment should not adversely affect adjacent joint function or otherwise restrict the climber's ability to assume essential positions.

\section{SYNERGISTIC TRUNK, LIMB, AND JOINT MYOFASCIAL SYSTEM ENHANCEMENT}

Muscle fibers are contractile components of a functional complex that is inseparable from its parallel and series non-contractile fibrous components. Activated muscle forces depend not only the anatomical structure of the muscle or muscle group, but also on the angle at which its fibers attach to intramuscular connective tissues, the epimysium and the deep fasciae (24). Fascia is an elastic tissue with established extensibility limits that effectuate motor coordination, movement perception and postural variation signaling (25). The endomysium, perimysium, epimysium and deep fasciae are fundamental to muscular force transmission, with each tissue possessing a specific role (24).

The myofascial system originates proximal to the upper and lower extremities to provide both joint and extremity protection, containment and stability. Neurophysiologists support the presence of a peripheral movement coordination system activated through gliding fascial layers (26). The brain interprets movement as three-dimensional agonistic synchrony during spatial movement task performance, not as independent muscle actions (27). Located within high muscular traction zones are densely innervated myofascial coordination centers (26). Through its basal tension, myofascial structures help maintain appropriate body posture. Acute injury or chronic inflammatory conditions at these centers can densify the tissue with unorganized collagen fibers, leading to pain. The body neutralizes this pain by adopting a compensatory, maladaptive posture to better
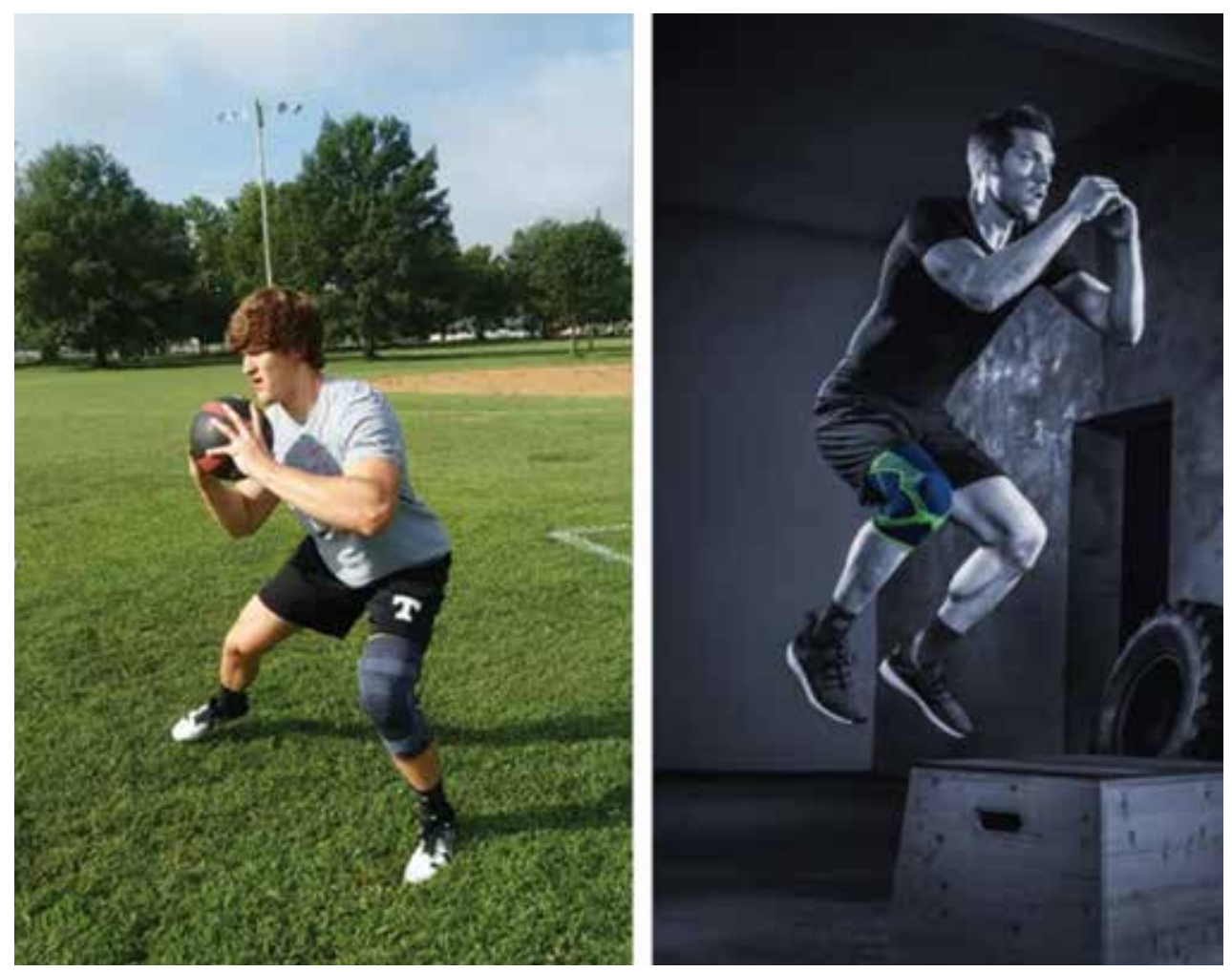

Figure 2. Evolving knee brace designs (A. Genu Medi Pro Knee Support; B. E + Motion Soft Support). 
re-establish basal tension. During healing, newly developed collagen fibers can only align themselves along the normal segmental force lines when normal basal myofascial tension exists (26). Myofascial system activation during movement can vary from individual motor units to multiple myofascial unit sequences across adjacent joint segments. To better engage myofascial system synergies, innovative protective and supportive garment or brace interfaces are being developed that blend compressive fit with embedded diagonal straps, mediolateral hinges, physiological monitoring (position, temperature, force, etc.) and region-specific capsuloligamentous, musculotendinous and myofascial augmentation to provide protection or support without over-constraint $(28,29)$ (figure 2). Many of these products attempt to combine the positive attributes of conventional garments and braces with athletic and kinesiotaping concepts that enhance proprioception/kinesthesia and natural myofascial system gliding function (figure 3). In association with this, fasciacytes produce hyaluronan to reduce friction, improving fascial mobility and decreasing myofascially-mediated pain (30). Stecco et al (31) reported that the gluteus maximus - fascia lata attachment was so large that the iliotibial tract was effectively its tendon of insertion. Thoracolumbar fascia forces can be transmitted from the gluteus maximus to the knee and from the latissimus dorsi to the shoulder. Painful patellar tendon conditions often occur from uncoordinated muscle or muscle group activation in the presence of anomalous myofascial tension (26). For these conditions, the treatment focus should not be at the local site of tendon pain, but rather it should be directed toward identifying the location of the myofascial incoordination (32). Myofascial continuity between the thoracolumbar fascia-abdominal muscles and the erector spinae - rectus abdominis also helps ensure dynamic postural trunk stability (33).

The supplemental limb and joint protection and support that used to be exclusively dependent on external brace use, now may at least partially be provided through innovative surgical internal bracing or supplemental extra-articular soft tissue repair (34). When feasible, surgical internal bracing may better facilitate capsuloligamentous tissue repair, concurrently retaining proprioceptive elements commonly lost during ligament reconstruction or joint replacement. Greater use of less rigid, functionally firm, protective and supportive garment or braces might be analogous to the contrasting properties of anatomical surgical repair versus reconstruction. Through more distributed surface contact area, these devices may enable better cutaneous, capsuloligamentous, musculotendinous and fascial mechanoreceptor function for dynamic joint stability than rigid braces, without reducing joint range of motion or impairing neuromuscular and neurovascular function. These

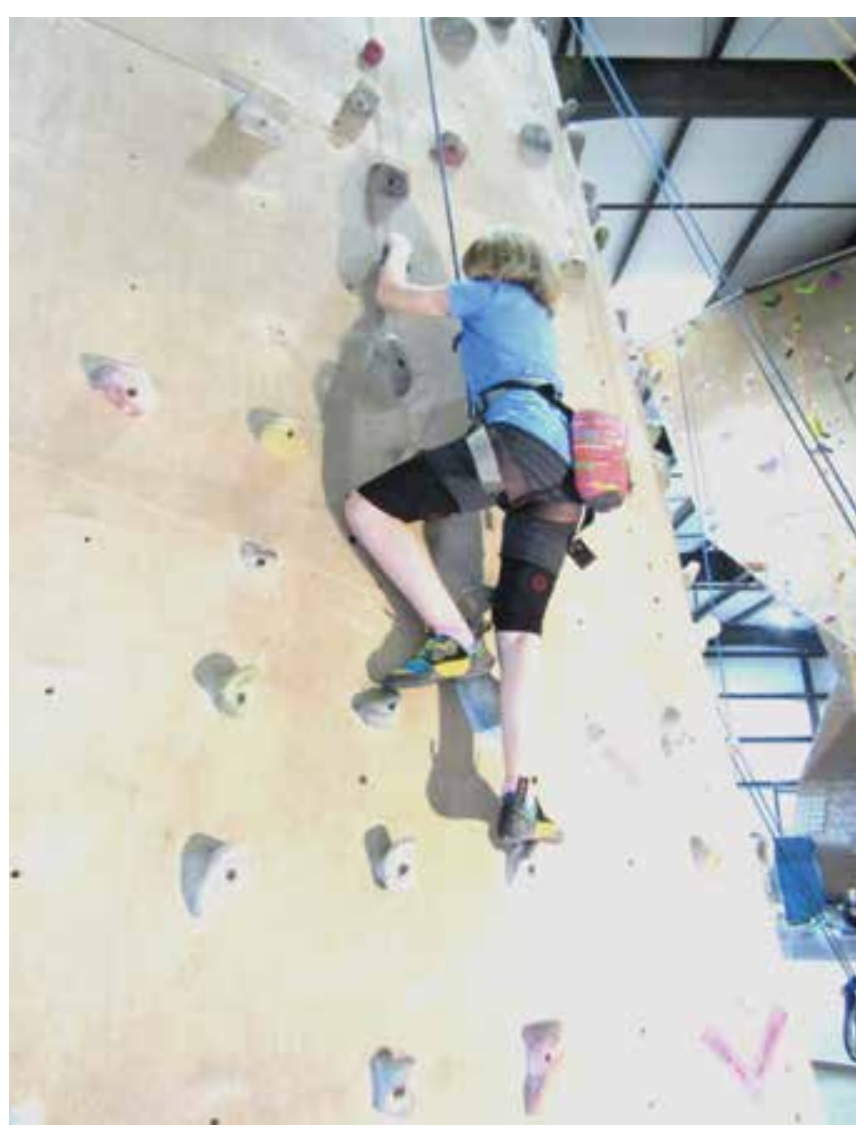

Figure 3. T-25 thigh compression garment to enhances dynamic knee control (CEP Topical Gear).

evolving supportive and protective garments or braces are being designed in concert with growing efforts to preserve joint health whenever feasible through more anatomical and biological surgical and non-surgical joint repair, rather than reconstruction. Impaired ACL injury neurosensory proprioception combined with ipsilateral rigid brace use may be related to the increasing frequency of contralateral, non-contact knee ACL injuries that have been observed. Use of regenerative biological healing progenitors such as stem cells, plasma-rich in platelets, or amnionic membrane tissue represents a developing and exciting contemporary healthcare sector. Innovations like these challenge rehabilitation clinicians to carefully determine the best combination of progressive joint loads, sport movement-based therapeutic exercises, and garment or brace use to accelerate both tissue healing and motor learning. This will ultimately result in more functionally responsive neuromuscular activation patterns and remodeled tissues that more closely match premorbid histology, morphology and biomechanical characteristics. 


\section{FOOTWEAR AND GLOVES}

The combination of feet pushing against the ground (figure 4) and the arms pulling generates holistic energy transfer throughout the entire body. Because of the close relationship between shoe-surface interactions and injury risk, footwear outsole configuration and its influence on locomotor traction is the most studied athletic footwear parameter (35). Cleats without studs provoke more vertical lower leg alignment at running stance phase initiation in combination with lower ankle and knee joint moments (36). Soft ground interfaces decrease foot movement in combination with increased ankle and knee moments (36). Extreme sport athlete footwear considerations should balance the increased primary and adjacent torsional joint injury risk

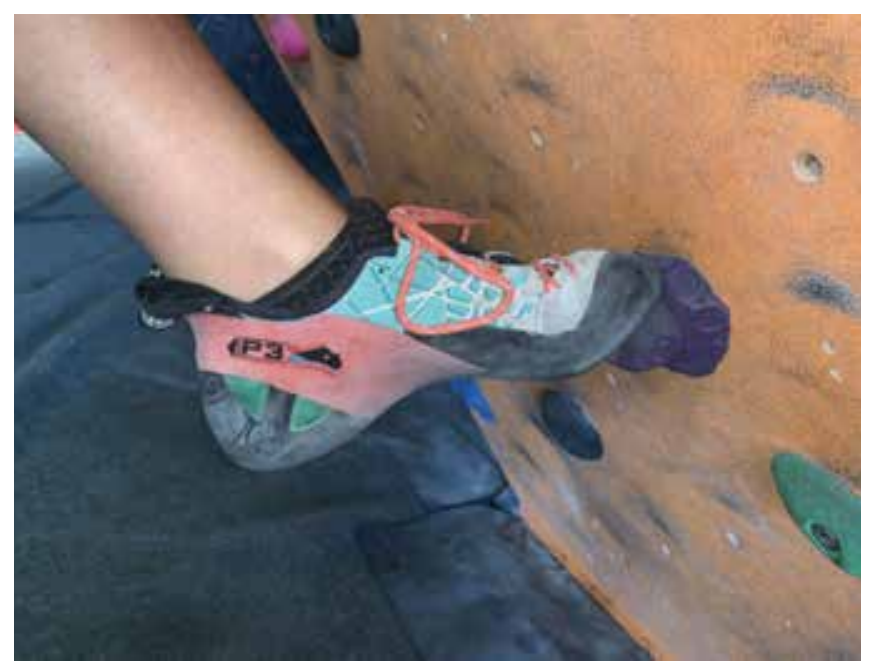

Figure 4. Climbing shoe - hold interface.
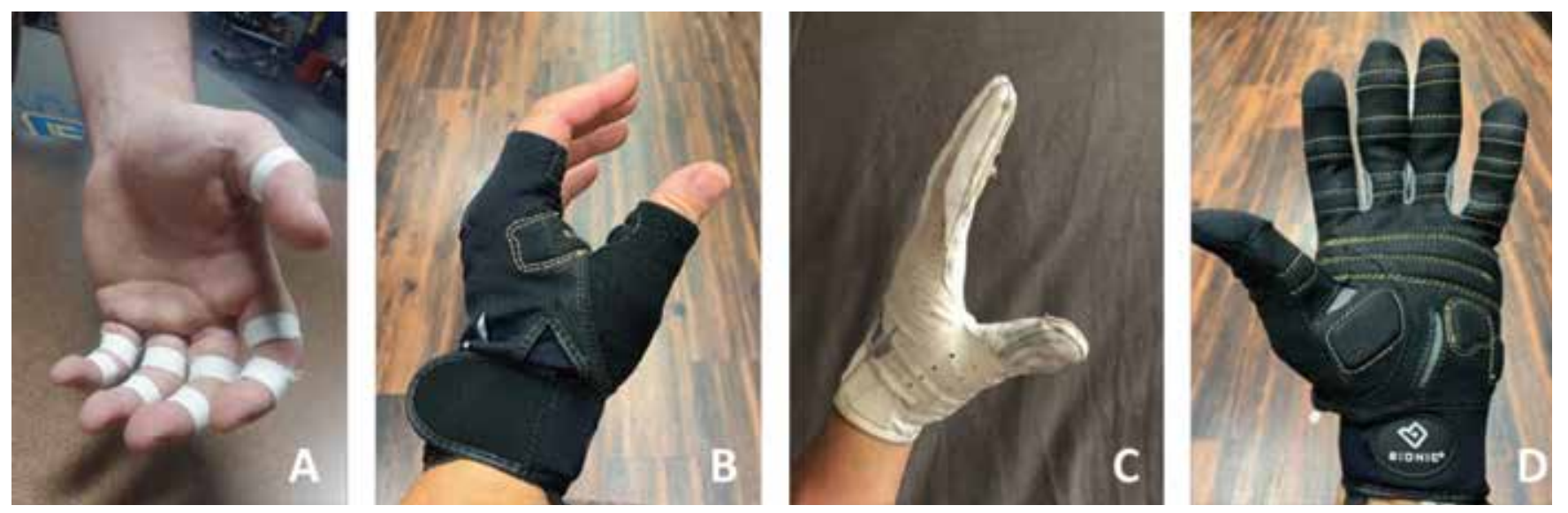

Figure 5. Hand-wrist supports. A. finger pulley taping; B. wrist and thumb stabilization; C. proprioceptive-gripping; D. full hand-wrist protection.

associated with footwear stud use and the potential for injury from increased slipping risk without their use $(35,36)$. Use of shorter, stubbier cleats have been recommended to prevent football knee injuries (37). Across a wide range of traditional and extreme sports, specialty gloves have been developed to enhance hand and wrist joint protection and support, touch perception, grip strength and proprioceptive awareness (figures 5). Sports that necessitate direct skin contact such as climbing often use more traditional athletic taping techniques to enhance joint stability without compromising fingertip sensory grip perception.

\section{EVOLVING PROTECTIVE AND SUPPORTIVE GARMENTS AND BRACES}

Bracing to protect or support, preserve and promote natural joint function is evolving from the use of heavier, rigid, over-constraining and poorly-fitting devices to lighter, more flexible, lower profile, function-enhancing garments or braces. This evolution is intended for three-dimensional protection, while not generating maladaptive primary or adjacent joint kinematics, or impaired/inhibited neuromuscular activation, neurovascular function or altered peak joint forces. Conceivably, protective and supportive garments or braces such as these may also provide a better foundation for enhancing cutaneous, capsuloligamentous, musculotendinous and myofascial system proprioception/kinesthesia and dynamic joint stability (figure 6). With a growing appreciation for the importance of peripheral, non-contractile joint stabilizers such as the anterolateral knee ligament (38), posterolateral and posteromedial corner knee and anteroinferior shoulder capsuloligamentous tissues (figure 7), rehabilitation clinicians are seeking better ways to enhance (1) 


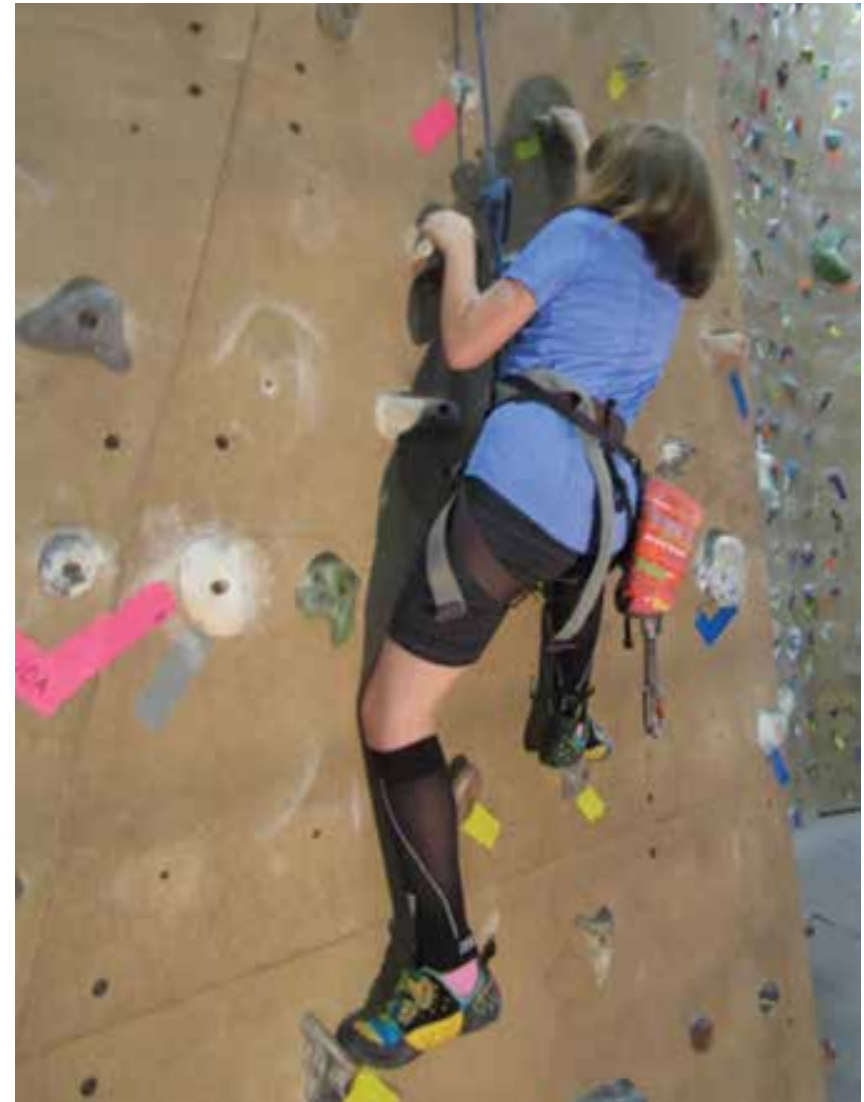

Figure 6. Wall climbing wearing compressive calf sleeves.

dynamic joint stability with improved patient comfort and use compliance. Athletic footwear has also evolved away from rigid, high motion control shoe designs to more preemptively protect and control the ankle, subtalar, transverse and longitudinal arches and first metatarsophalangeal joints, without the overconstraint that transfers loading forces proximally to the knee, hip and low back. The growing use of low movement control shoes in an attempt to modify running mechanics toward more forefoot-directed contact patterns with greater intrinsic core foot muscle use may help mitigate the influence of excessive, poorly controlled knee and hip joint loading forces.

Sleeve-based protective and supportive garments or braces have the advantage of simpler, quicker application, avoidance of piston-like positional changes that lead to slippage during joint movements and easier adaptation to neuromuscular inhibition/atrophy and activation recovery/hypertrophy limb girth changes. Protective and supportive garments or braces can also be more easily integrated into compressive sport shirts, shorts and leggings with or without additional orthosis use $(28,29)$.
Therapeutic exercises that combine task-specific self-efficacy and problem-solving skill development may best optimize innovative protective and supportive garment or brace use. Given the variety of movements that come under the extreme sport definition, appropriate therapeutic exercise selection or creation provides an ideal method for the athlete to learn from, "if this, then that," situations and scenarios to simulate the sport-specific movements needed to optimize joint specific, regional, and whole body coordinated neuromuscular, sensorimotor, and vestibular system protective responses. Well-designed movement-based task- and sport-specific therapeutic exercise programs should more effectively transition from higher level, executive cognitive function with careful attention to developing movement quality and form to lower level, automatic responses

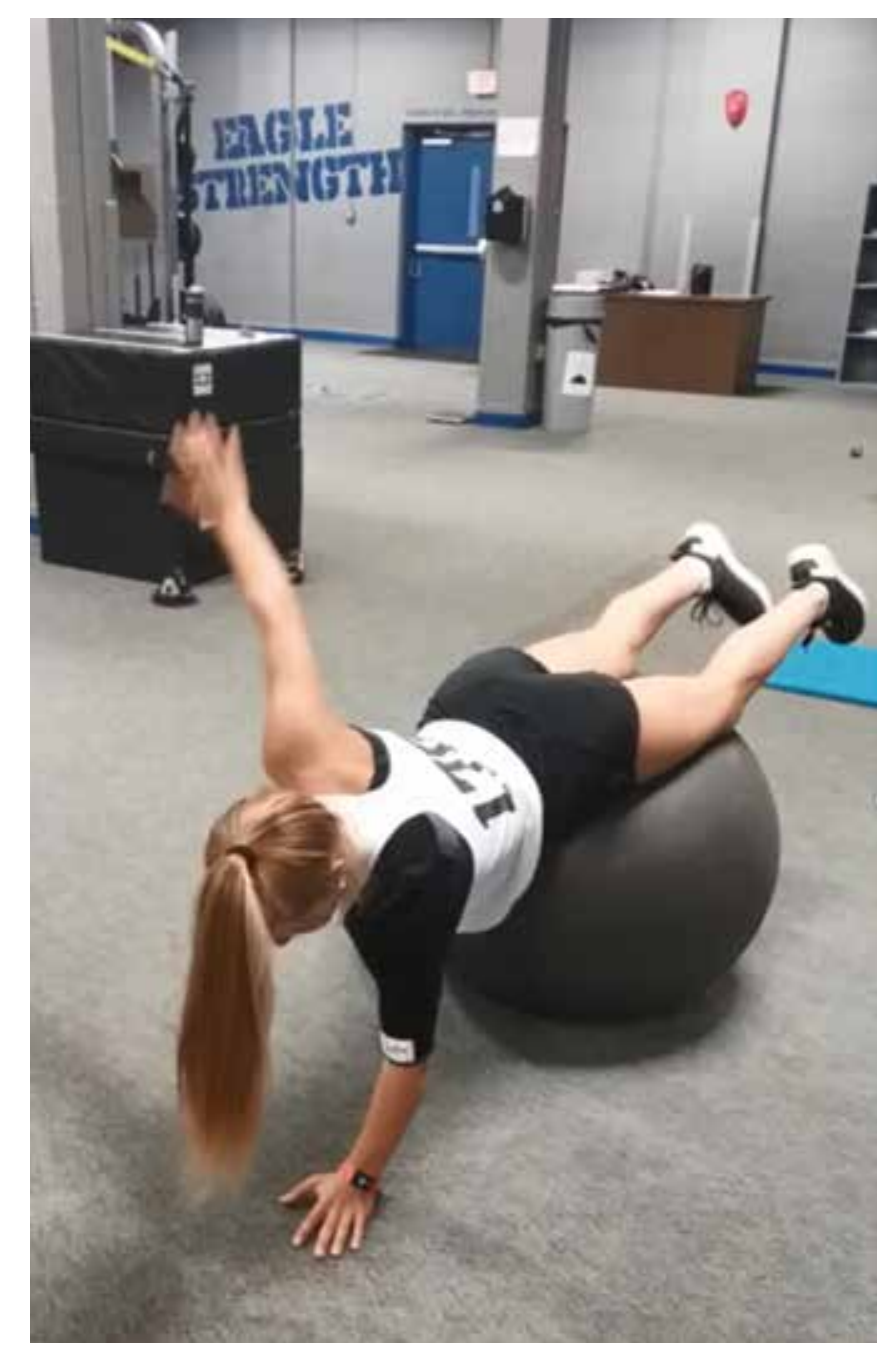

Figure 7. Glenohumeral joint support using the DonJoy Sully Shoulder Stabilizer. 
as the athlete is confronted with random perturbations or unexpected chaos. To better enable healing tissue remodeling, whenever feasible, the extreme sport athlete should periodize protective or supportive garment or brace use. As with the stress shielding of cast immobilization and limited weight bearing crutch use, biomechanically competent tissue healing only promotes tissues remodel in response to progressively greater natural joint loading conditions.

Rigid braces will always have a vital role in athletic rehabilitation and recovery, such as when repetitive or higher energy contact or collision risks can be expected (downhill skiing, American football) or when high joint movement or alignment constraints are needed. These supports may also be the ideal intervention with treating the extreme sport athlete who has sustained a severe multi-ligament bi-cruciate or cruciate-corner knee injury, when natural joint kinematics have not been precisely restored, when articular

\section{REFERENCES}

1. Collins L, Collins D. Professional judgement and decision-making in adventure sports coaching: the role of interaction. J Sports Sci 2016;34(13):1231-1239.

2. Stecco A, Stern R, Fantoni I, De Caro R, Stecco C. Fascial disorders: Implications for treatment. PM R 2016;8(2):161168.

3. Lichtenstein MB, Larsen KS, Christiansen E, Stoving RK, Bredahl TVG. Exercise addiction in team sport and individual sport prevalences and validation of the exercise addition inventory. Addict Res Theory 2014;22:431-437.

4. Buckley RC. Adventure thrills are addictive. Front Psychol 2015;6:1915.

5. Schoffl V, Morrison A, Schwarz U, Schoffl I, Kupper T. Evaluation of injury and fatality risk in rock and ice climbing. Sports Med 2010;40(8):657-679.

6. Magiera A, Roczniok R, Sadowaska-Krepa E, Kempa K, Placek $\mathrm{O}$, Mostowik A. The effect of physical and mental stress on the heart rate cortisol and lactate concentrations in rock climbers. J Hum Kinetics 2018;65:111-123.

7. Hodgson CI, Draper N, McMorris T, Jones G, Fryer S, Coleman I. Perceived anxiety and plasma cortisol concentrations following rock climbing with differing safety rope protocols. Br J Sports Med 2009;4:531-535.

8. Clemente-Suarez VJ, Robles-Perez JJ, Fernandez-Lucas J. Psycho-physiological response in an automatic parachute jump. J Sports Sci 2017;35(19):1872-1878.

9. Allen-Collinson JA, Crust L, Swann C. 'Endurance work': Embodiment and the mind-body nexus in the physical culture of high-altitude mountaineering. Sociology 2018;52(6):1324-1341.

10. Seifert L, Orth D, Button C, Brymer E, Davids K. An ecological dynamics framework for the acquisition of perceptual-motor skills in climbing. In: Extreme Sports Medicine, (ed) F. Feletti (Springer International Publishing, pp 365-382, 2017).

11. Papacosta E, Nassis GP. Saliva as a tool for monitoring steroid, peptide and immune markers in sport and exercise science. J Sci Med Sport 2011;14(5):424-434. incongruency (step-off) remains, when osteoarthritic joint changes exist, or when chondral surface repair or reconstruction warrants off-loading or re-alignment osteotomy.

\section{SUMMARY}

Through greater surface contact area and enhanced cutaneous, capsuloligamentous, musculotendinous and myofascial system mechanoreceptor function, a new evolution of protective and supportive trunk, limb and joint garments or braces may more closely simulate the subtle, sequential natural protection and proprioception/kinesthesia and dynamic joint stability

\section{CONFLICT OF INTERESTS}

The authors declare that they have no conflict of interests (39).

12. Suay F, Salvador A. Cortisol. In: Psychoneuroendocrinology of sport and exercise. Foundations, markers, trends. Ehrlenspiel F, Strahler K (eds), Routledge, Abingdon, 2012.

13. Sperich B, Achtzehn S, Buhr M, Zinner Ch, Zelle S, Holmberg $\mathrm{HCh}$. Salivary control, heart rate, and blood lactate responses during elite downhill mountain bike racing. Int J Sports Physiol Perform 2012;7:47-52.

14. Lindinger MI, Kowalchuk JM, Heigenhauser GJF. Applying physiochemical principles to skeletal muscle acidbase status. Am J Physiol Regul Integr Comp Physiol 2005;289(3):891-894.

15. Gladden LB. Lactate metabolism: A new paradigm for the third millennium. J Physiol 2004;558(1):5-30.

16. Sheel AW, Seddon N, Knight A, McKenzie DC, Warburton DE. Physiological responses to indoor rock-climbing and their relationship to maximal cycle ergometry. Med Sci Sports Exerc 2003;35(7):1225-1231.

17. Espana-Romero V, Ortega Porcel FB, Artero EG, Jimenez-Pavron D, Gutierrez Sainz A, Castillo Garzon MJ, Ruiz JR. Climbing time to exhaustion is a determinant of climbing performance in high-level sport climbers. Eur J Appl Physiol 2009;107(5):517-525.

18. Michailov ML. Workload characteristic, performance limiting factors and methods for strength and endurance training in rock climbing. Med Sport 2014;18(3):97-106.

19. Bertuzzi RC, Franchini E, Kokubun E, Kiss MA. Energy system contributions in indoor rock climbing. Eur J Appl Physiol 2007;101(3):293-300.

20. Watts PB, Drobish KM. Physiological responses to simulated rock climbing at different angles. Med Sci Sports Exerc 1998;30:1118-1122.

21. Seifert L, Boulanger J, Orth D, Davids K. Environmental design shapes perceptual-motor exploration, learning and transfer in climbing. Front Psychol 2015;6:1819.

22. Kostrubiec V, Zanone PG, Fuchs A, Kelso JAS. Beyond the blank slate: Routes to learning new coordination patterns depend on the intrinsic dynamics of the learner: Experimen- 
tal evidence and theoretical model. Front Hum Neurosci 2012;6:222.

23. Orth D, Davids K, Chow J-Y, Brymer E, Seifert L. Behavioral repertoire influences the rate and nature of learning in climbing: Implications for individualized learning designs in preparation for extreme sports participation. Front Psychol 2018;9:949.

24. Turrina A, Martínez-González MA, Stecco C. The muscular force transmission system: role of the intramuscular connective tissue. J Bodyw Mov Ther 2013;17(1):95-102.

25. Gray H. Anatomia. Ed. Zanichelli, Bolgna, Italy, 1993.

26. Stecco L. Fascial manipulation for musculoskeletal pain. Piccin Nuova Libraria S.p.A., Padova, Italy, 2004.

27. Rizzolatti G, Luppino G. The cortical motor system. Neuron 2001;31:889-901.

28. Scataglini S, Andreoni G, Truyen E, Warnimont L, Gallant J, Van Tiggelen D. Design of smart clothing for Belgian soldiers through a preliminary anthropometric approach. In: Proceedings 4th DHM Digital Human Modeling: Montreal, Quebec, Canada, June 15-17, 2016.

29. Scataglini S, Andreoni G, Gallant J. Smart clothing design issues in military applications. In: AHFE 2018, AISC 795 (Ed) T. Abram, pp 1-11, 2019.

30. Stecco C, Fede C, Macchi V, Porzionato A, Petrelli L, Biz C, Stern R, De Caro R. The fasciacytes: A new cell devoted to fascial gliding regulation. Clin Anat 2018;31(5):667-676.

31. Stecco A, Gilliar W, Hill R, Fullerton B, Stecco C. The anatomical and functional relation between gluteus maximus and fascia lata. J Bodyw Mov Ther 2013;17(4):512-517.

32. Pedrelli A, Stecco C, Day JA. Treating patellar tendinopathy with Fascial Manipulation. J Bodyw Mov Ther 2009;13(1):73-80.
33. Fan C, Fede C, Gaudreault N, Porzionato A, Macchi V, DE Caro R, Stecco C. Anatomical and functional relationships between external abdominal oblique muscle and posterior layer of thoracolumbar fascia. Clin Anat 2018;31(7):10921098.

34. Mackay GM, Blyth MJ, Anthony I, Hopper GP, Ribbans WJ. A review of ligament augmentation with the InternalBrace $^{\mathrm{TM}}$ : The surgical principle is described for the lateral ankle ligament and ACL repair in particular, and a comprehensive review of other surgical applications and techniques is presented. Surg Technol Int 2015;26:239-255.

35. Kulessa DJ, Gollhofer A, Gehring D. The influence of football shoe characteristics on athletic performance and injury risk - a review. Footwear Science 2017; doi: 10.1080/ 19424280.2017.1284273.

36. Müller C, Sterzing T, Lake M, Milani TL. Different stud configurations cause movement adaptations during a soccer turning movement. Footwear Science 2010; doi: 10.1080/ 19424281003685702.

37. McGinty JC. What's the stress test for NFL cleats? The right shoe can help prevent lower limb traumas, which account for $60 \%$ of league injuries. Wall Street Journal (Online): New York, NY, 2018.

38. Macchi V, Porzionato A, Morra A, Stecco C, Tortorella C, Menegolo M, Grignon B, De Caro R. The anterolateral ligament of the knee: a radiologic and histotopographic study. Surg Radiol Anat 2016;38(3):341-348.

39. Padulo J, Oliva F, Frizziero A, Maffulli N. Muscles, Ligaments and Tendons Journal - Basic principles and recommendations in clinical and field Science Research: 2018 update. MLTJ 2018; 8(3): 305 - 307. 